\title{
Static Force Balancing of a 2R1T Parallel Manipulator with Remote Center of Motion
}

\author{
Abdullah Yaşır ${ }^{1[0000-0001-5230-5883]}$, Gökhan Kiper ${ }^{20000-0001-8793-724 X]}$, M.İ. Can Dede 2 [0000- \\ 0001-6220-6678] and Volkert van der Wijk ${ }^{1[0000-0002-7953-4368]}$ \\ ${ }^{1}$ Delft University of Technology, Mekelweg 2, 2628 CD, Delft, The Netherlands \\ ${ }^{2}$ Izmir Institute of Technology, Gülbahçe, 35430, Izmir, Turkey
}

\begin{abstract}
Assistive robots in surgical applications should be gravity balanced due to safety considerations. This study presents a gravity balancing solution for a 3-degree-of-freedom parallel manipulator to be used as an endoscope navigation robot for transnasal minimal invasive surgery applications. The manipulator has a rather simple structure that allows individual balancing of the three legs in their respective planes of motion. First, sole counter-mass balancing is investigated, but it is seen that the extra mass amount is too much. Sole spring balancing is not considered as an option due to constructional complexity. A hybrid solution as a combination of counter-mass and spring balancing is devised. In the proposed solution, the masses on the distal links of a leg are balanced with counter-masses so that all masses are lumped to the link connected to the base of the manipulator. Hence the problem is simplified into the balancing of a pendulum. The necessary formulations are derived and numerical calculations demonstrate that the hybrid balancing yields a feasible solution.
\end{abstract}

Keywords: Static Balancing, Parallel Manipulator, Remote Center of Motion.

\section{$1 \quad$ Introduction}

In applications such as cutting or welding of circular objects, teleoperation, radiotherapy, imaging, exoskeletons and minimal invasive surgery, manipulators with remote center of motion (RCM) are frequently utilized [Teichgräber, Müglitz, Berger, 2017; Schena, 2007]. RCM is a point where one or more rotational and translational movements are pivoted outside the mechanism so that the mechanism needs no joint at that point. Geometrically, a motion with an RCM describes the motion of a line constrained to pass through a fixed point in space. For a manipulator with RCM, the endeffector may have up to 4 degrees-of-freedom (dof) (3 rotational + 1 translational dof) through the RCM point: pitch, yaw, roll and heave motions (Fig. 1). If the endeffector pivotted on the RCM is a cylindrical tool such as welding torch or endoscope, the roll motion may not be required. This study deals with a parallel manipulator with pitch, yaw and heave movements which define a $2 \mathrm{R} 1 \mathrm{~T}$ (R: rotation; $\mathrm{T}$ : translation) motion pattern where the translation direction is perpendicular to the plane defined by two orthogonally intersecting rotation axes. The intersection point of the rotation axes 
is the RCM. In such an application the end-effector should purely have the $2 \mathrm{R} 1 \mathrm{~T}$ motion and parasitic motions are not allowed.

The structural synthesis of 3-dof parallel manipulators with 2R1T motion pattern and RCM is recently presented by Yaşır and Kiper (2017). Among several manipulator architecture alternatives, the 2URRR-URR parallel manipulator is selected as an endoscope holder for a minimally invasive transnasal pituitary gland surgery application. The kinematic diagram of the mechanism is given in Fig. 1, where pitch, yaw and heave motions constituting a $2 \mathrm{R} 1 \mathrm{~T}$ motion pattern are indicated by red color. These three motions are sufficient for endoscope movements (Tanigucci et al., 2010). The kinematic and constructional design of the manipulator is described in (Yaşır, 2018). The URR leg is the middle leg while the two URRR legs are the side legs. Except the $\mathrm{R}$ joints along endoscope axis along $\mathrm{DE}$, all legs have the same kinematic structure as the URR structure, which contain 3 revolute joints with parallel axes. All legs move on a respective plane and the angle of each leg plane can be changed by a revolute joint whose axis is within that plane (axes along $A_{0} D, B_{0} d$ and $C_{0} D$ ).

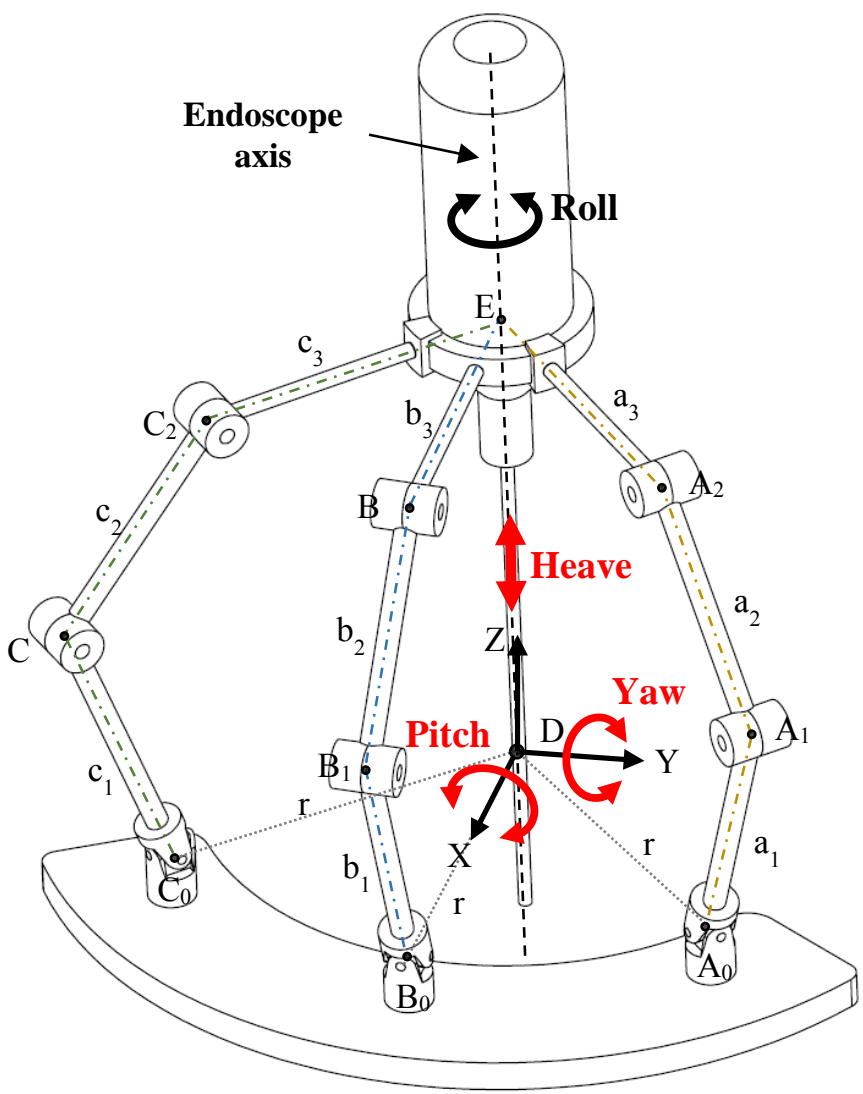

Fig. 1. Kinematic diagram of the 2URRR-URR parallel manipulator with remote center of motion D about which the platform has 2 rotations and 1 translation (Yaşır, 2018). 
In applications where actuation due to gravitational forces is undesired, static balance techniques are applied. This means that no actuation is needed to keep the manipulator steady at any pose. Static balance can be achieved by applying counter-masses and springs (Wang, Gosselin, 1999-2000; Baradat et al., 2008; Van der Wijk, 2014; Herder, 2001). A common method for balancing a parallel manipulator is leg-by-leg balancing where each leg is considered individually as a serial chain and the mass of the platform is distributed mass equivalently among the legs [Wang, Gosselin, 19992000; Van der Wijk, 2014]. One major problem with balancing serial chains is that the counter-masses generally become extremely heavy, especially the last one at the link with the base (Van der Wijk, 2009). Balancing with springs is a lightweight solution however implementing springs in serial chains results into complex constructions (Herder, 2001). Since for each spring a vertical reference link is needed, in a serial chain this leads to at least one parallelogram linkage per dof.

The goal of this paper is to show a practical static balance solution for the 2URRRURR manipulator comprising a specific combination of counter-masses and springs. This results in a relatively low mass and low complex solution. First the common way of balancing a leg with solely counter-masses is shown. Subsequently the function of one of the counter-masses is taken over by a spring for which the balance conditions are derived. Finally a numerical study is presented as an example.

\section{Common method to balance a leg with counter-masses}

First, the case in which only counter-masses are used for static balancing is investigated in order to see whether it is feasible or not. The masses of the links are neglected and the end-effector payload is equally distributed to the three legs and the distributed masses are lumped at the end of the distal link in each leg.

In Fig. 2, a diagram for counter-mass balancing of one of the legs in the mechanism is given. All three legs have the same structure. The payload for the leg is $\mathrm{m}_{\mathrm{c}}$, which is one-third of the mass of the end effector. $M_{a}, M_{b}$ and $M_{c}$ are the countermasses and $b_{a}, b_{b}$ and $b_{c}$ are their extension lengths from the joints $A_{0}, A$ and $C$, respectively. Parameters $r_{a}, r_{b}$ and $r_{c}$ are the link lengths. $\delta$ is the angle of the base of the mechanism with respect to the ground horizontal level. $\eta_{1}$ and $\eta_{2}$ are the angle limits for the proximal link of the leg. For the specific problem at hand, the payload of the mechanism is $1,2 \mathrm{~kg}$ in total. The payload consists of a platform and an endoscope attached to the platform. So, for a leg, $m_{c}=400$ gr. Also, $\eta_{1}=57,2^{\circ}$ and $\eta_{2}=209,7^{\circ}$ are taken from the kinematic design study according to the desired workspace specifications. The counter-masses can be found using the following equations:

$$
\begin{gathered}
M_{c} b_{c}=m_{c} r_{c} \Rightarrow M_{c}=\frac{m_{c} r_{c}}{b_{c}} \\
M_{b} b_{b}=\left(m_{c}+M_{c}\right) r_{b} \Rightarrow M_{b}=\frac{\left(m_{c}+M_{c}\right) r_{b}}{b_{b}} \\
M_{a} b_{a}=\left(m_{c}+M_{c}+M_{b}\right) r_{a} \Rightarrow M_{a}=\frac{\left(m_{c}+M_{c}+M_{b}\right) r_{a}}{b_{a}}
\end{gathered}
$$




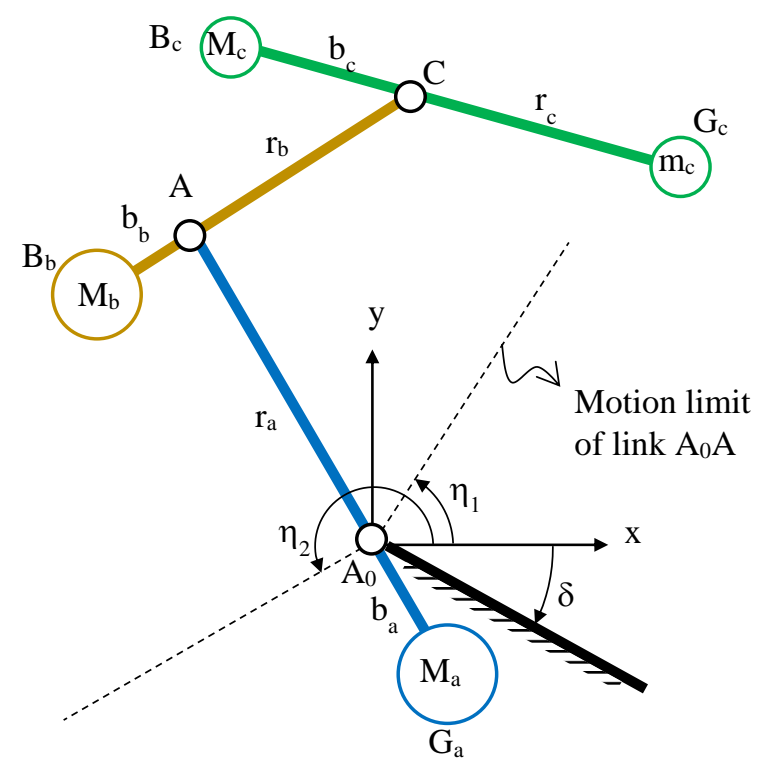

Fig. 2. Common static force balance solution of a leg of three links in series with countermasses. The center of mass of the leg is stationary in base pivot $A_{0}$

Counter-mass distances $b_{a}, b_{b}, b_{c}$ are selected considering link collisions and the footprint of the mechanism. The link parameters are set according to the kinematic design and the counter-masses are calculated (see Table 1).

Table 1. Mass balancing parameters for one leg $\left(\mathrm{m}_{\mathrm{c}}=400 \mathrm{gr}\right)$

\begin{tabular}{|c|c|c|c|c|c|}
\hline \multicolumn{2}{|c|}{ Link Length $(\mathrm{mm})$} & \multicolumn{2}{c|}{ Extension Length $(\mathrm{mm})$} & \multicolumn{2}{c|}{ Mass $(\mathrm{gr})$} \\
\hline $\mathrm{r}_{\mathrm{a}}$ & 135 & $\mathrm{~b}_{\mathrm{a}}$ & 100 & $\mathrm{M}_{\mathrm{a}}$ & 3712,5 \\
\hline $\mathrm{r}_{\mathrm{b}}$ & 195 & $\mathrm{~b}_{\mathrm{b}}$ & 80 & $\mathrm{M}_{\mathrm{b}}$ & 1950 \\
\hline $\mathrm{r}_{\mathrm{c}}$ & 200 & $\mathrm{~b}_{\mathrm{c}}$ & 200 & $\mathrm{M}_{\mathrm{c}}$ & 400 \\
& \multicolumn{2}{|}{} & $\mathbf{M}_{\text {total }}$ & 6062,5 \\
\hline
\end{tabular}

As a result of this study, approximately $6 \mathrm{~kg}$ additional mass for one leg and totally 18 $\mathrm{kg}$ additional mass is needed to balance $1,2 \mathrm{~kg}$ payload even though the masses of the links are neglected. A balancing with that amount of mass is not practical since the mechanism needs to be compact and lightweight. Also, due to the motion range of the proximal link, there may be some collisions with the manipulator's base.

\section{Low mass and low complex solution by introducing a spring}

A hybrid static force balance solution is proposed by which function of the largest counter-mass $\left(\mathrm{M}_{\mathrm{a}}\right)$ in Fig. 2 is taken over by a spring. This solution is illustrated in Fig.3, where this time, the link masses $m_{a}, m_{b}$ and $m_{c}$ are also taken into account. The counter-mass $M_{c}$ balances masses $m_{\text {pay }}$ and $m_{c}$, whereas counter-mass $M_{b}$ balances 
masses $m_{\text {pay }}$ and the total mass $M_{c}+m_{\text {pay }}+m_{c}$. The equivalent mass $M_{a}=m_{a}+m_{b}+$ $\mathrm{m}_{\mathrm{c}}+\mathrm{m}_{\text {pay }}+\mathrm{M}_{\mathrm{b}}+\mathrm{M}_{\mathrm{c}}$ is balanced by a linear spring.

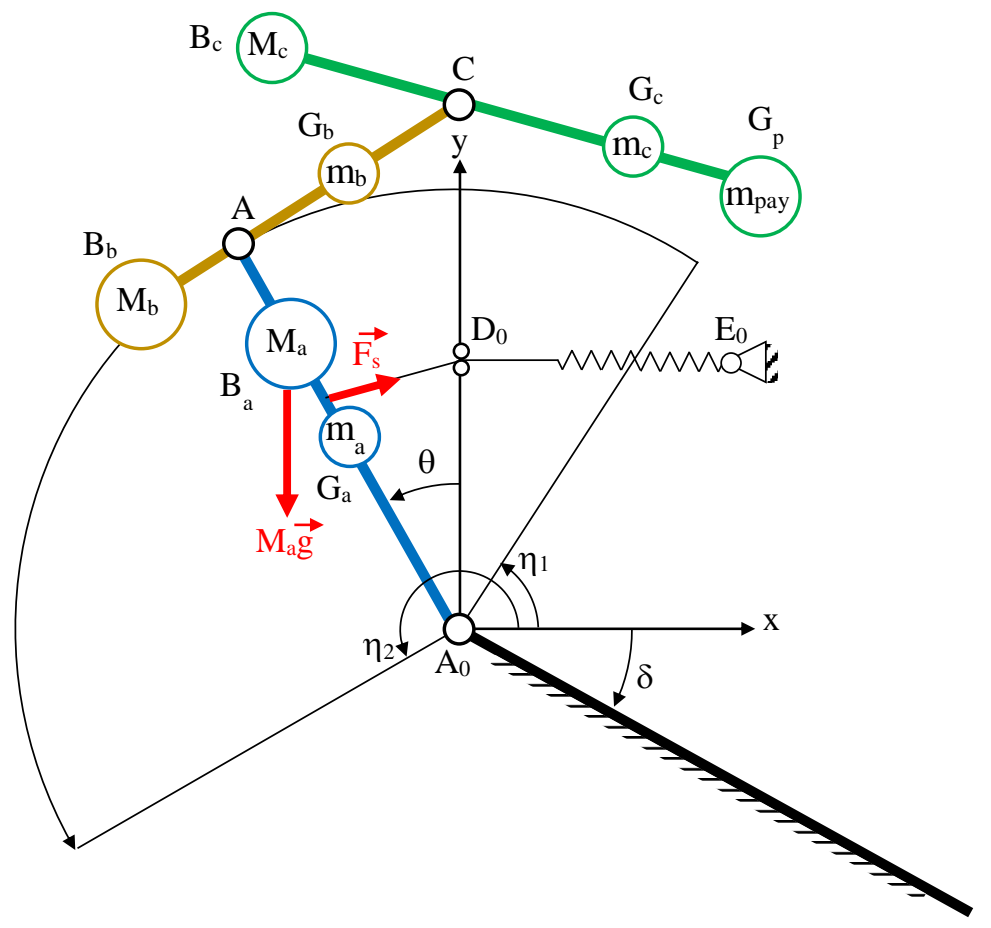

Fig. 3. Diagram for the hybrid static force balancing of a leg with three links in series and with two counter-masses and a spring

The counter-masses $\mathbf{M}_{\mathrm{c}}$ and $\mathrm{M}_{\mathrm{b}}$ in Fig. 3 are computed by formulations similar to (1) and (2), but also considering the link masses and locations. However the counter-mass $M_{a}$ is not the same mass as $M_{a}$ in Fig. 2. It is the total leg mass and $g_{a, t}=\left|A_{0} B_{a}\right|$ is the total CoM location of the whole leg. Also, $m_{\text {pay }}$ is the payload for a leg. Let $\left|A_{0} G_{a}\right|=$ $\mathrm{g}_{\mathrm{a}},\left|\mathrm{AG}_{\mathrm{b}}\right|=\mathrm{g}_{\mathrm{b}},\left|\mathrm{CG}_{\mathrm{c}}\right|=\mathrm{g}_{\mathrm{c}}$ and $\left|\mathrm{CG}_{\mathrm{p}}\right|=\mathrm{g}_{\text {pay }}$ denote the locations of the masses of links $\mathrm{a}$, b, c and payload. So,

$$
\begin{gathered}
M_{a}=m_{a}+m_{b}+m_{c}+m_{p a y}+M_{b}+M_{c} \\
g_{a, t}=\frac{\left(m_{b}+m_{c}+m_{p a y}+M_{c}+M_{b}\right) r_{a}+m_{a}+g_{a}}{b_{a}}
\end{gathered}
$$




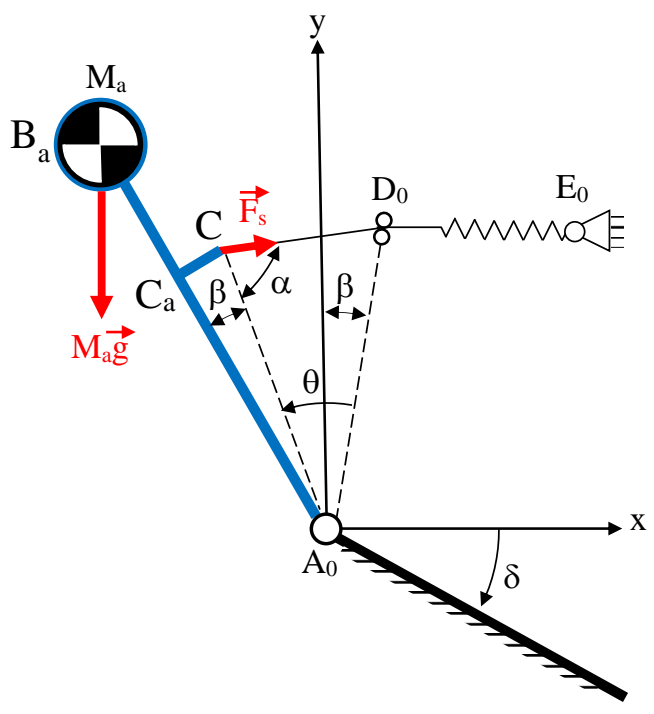

Fig. 4. Diagram for a basic gravity equilibrator using a zero-free length spring and a pulley

$$
\text { (Herder, 2001) }
$$

When all masses in Fig. 3 are reduced to the $\mathrm{A}_{0} \mathrm{~A}$ link, the problem simplifies to balancing of a pendulum also known as a basic gravity equilibrator as in Fig. 4. In Fig. 4, also an angle $\beta$ is introduced, which enables constructional simplicity. This angle has no effect in the calculations and without loss of generality it can be assumed $\beta=0$ (Herder, 2001). Let $\left|A_{0} D_{0}\right|=\left|A_{0} C\right|=f$, where $D_{0}$ is a fixed point for the string attached to the spring. Using sine theorem in triangle $\mathrm{A}_{0} \mathrm{CD}_{0}$ :

$$
\frac{f}{\sin \alpha}=\frac{\left|\mathrm{CD}_{0}\right|}{\sin \theta} \Rightarrow\left|\mathrm{CD}_{0}\right|=\frac{f \sin \theta}{\sin a}
$$

$\left|\mathrm{D}_{0} \mathrm{E}_{0}\right|$ length and length of the string attached to the spring depend on the free length $\mathrm{l}_{0}$ of the spring and should be evaluated such that the spring is load-free when $\mathrm{B}_{\mathrm{a}}$ is on the $y$-axis, i.e. when the pendulum is in vertical position. Also, balancing error due to the wire wrapped around pulley is neglected since the size of the pulley is relatively small. Examples for reducing this error is given by Barents et al. (2009). Let $\mathrm{k}$ be the spring constant, and $\mathrm{s}=\left|\mathrm{CD}_{0}\right|$ is the effective spring displacement. Then,

$$
F_{s}=k s
$$

Using Eqs. (6), (7) and moment equilibrium for $\mathrm{M}_{\mathrm{a}}$ about $\mathrm{A}_{0}$ :

$$
\begin{gathered}
g_{a, t} M_{a} g \sin \theta=f F_{s} \sin \alpha=f k s \sin \alpha=f^{2} k \sin \theta \\
\Rightarrow k=\frac{g_{a, t} M_{a} g}{f^{2}}
\end{gathered}
$$


After these formulations, a numerical study is carried out and results are tabulated in Table. 2. In this study, the values of the link masses and their distances to the proximal links are taken from a CAD model. Most of the mass of the platform is carried by the distal link of the link 3. The remaining payload (i.e. endoscope group), $\mathrm{m}_{\text {pay }}{ }^{\text {tot }}$ is distributed to legs in various ratios such that the same spring can be used for the whole legs. Therefore, for the distribution ratios of 0,4206 and 0,1588 for the side legs and the middle leg, respectively, and $\mathrm{f}=78 \mathrm{~mm}$, a spring with $\mathrm{k}=0,5161 \mathrm{~N} / \mathrm{mm}$ is determined to be used for each leg. As a result, hybrid balancing method reduced the total mass approximately from $18 \mathrm{~kg}$ to $7 \mathrm{~kg}$. When compared to complete mass balancing case, the hybrid balancing solution is more practical to implement.

Table 2. Hybrid balancing parameters

\begin{tabular}{|c|c|c|c|c|c|c|c|c|}
\hline & & $g r$ & & $m m$ & & $\mathrm{~mm}$ & & $g r$ \\
\hline \multirow{5}{*}{ } & $\mathbf{m}_{\mathbf{a}}$ & 43,5 & $\mathbf{g}_{\mathbf{a}}$ & 79,5 & $\mathbf{g}_{\mathrm{a}, \mathrm{t}}$ & 134 & $\mathbf{M a}_{\mathbf{a}}$ & 2388,9 \\
\hline & $\mathbf{m}_{\mathbf{b}}$ & 47,4 & $\mathbf{g}_{\mathbf{b}}$ & 117,8 & $\mathbf{b}_{\mathbf{b}}$ & 80 & $\mathbf{M}_{\mathbf{b}}$ & 1649,8 \\
\hline & $\mathbf{m}_{\mathbf{c}}$ & 63,2 & $\mathbf{g}_{\mathbf{c}}$ & 90,7 & $\mathbf{b}_{\mathbf{c}}$ & 200 & $\mathbf{M}_{\mathbf{c}}$ & 293,8 \\
\hline & $\mathbf{m}_{\text {pay }}$ & 291,2 & gay & 182,1 & & & & \\
\hline & $\mathbf{m}_{\mathrm{c}, \mathrm{t}}$ & 354,4 & $\mathrm{~g}_{\mathrm{c}, \mathrm{t}}$ & 165,8 & & & & \\
\hline \multirow{5}{*}{ 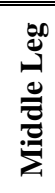 } & $\overline{m_{a}}$ & 53 & $\mathrm{ga}$ & 69,1 & $\mathbf{g}_{\mathrm{a}, \mathrm{t}}$ & 133,5 & $\mathbf{M a}_{\mathbf{a}}$ & 2397,0 \\
\hline & $\mathbf{m}_{\mathbf{b}}$ & 54,1 & $\mathbf{g}_{b}$ & 112,7 & $\mathbf{b}_{\mathbf{b}}$ & 80 & $\mathbf{M}_{b}$ & 1568,0 \\
\hline & $\mathbf{m}_{\mathrm{c}}$ & 314 & $\mathbf{g}_{\mathbf{c}}$ & 126,0 & $\mathbf{b}_{\mathbf{c}}$ & 200 & $\mathbf{M}_{\mathrm{c}}$ & 298,0 \\
\hline & $\mathbf{m}_{\text {pay }}$ & 109,9 & gpay & 182,1 & & & & \\
\hline & $\mathbf{m}_{\mathrm{c}, \mathrm{t}}$ & 423,9 & $\mathbf{g}_{\mathrm{c}, \mathrm{t}}$ & 140,6 & & & & \\
\hline & & & & & & & $\mathbf{M}_{a}{ }^{\text {tot }}$ & 7174,8 \\
\hline
\end{tabular}

\section{Conclusions}

A gravity balancing solution for a 3-dof parallel manipulator is presented. Due to high additional amount of masses, solely counter-mass balancing is not feasible, whereas solely spring balancing is not feasible due to constructional complexity. Instead, a hybrid solution is devised as a combination of counter-mass and spring balancing. Calculations demonstrate that the hybrid solution yields much less extra mass, whereas construction seems feasible. The solution devised in this paper will be applied first in constructional design level and then via a prototype as soon as several other design issues about the related application are addressed. 


\section{Acknowledgments}

This work is supported by The Scientific and Technological Research Council of Turkey via grant number $116 \mathrm{M} 272$.

\section{References}

1. Teichgräber, C., Müglitz, J. and Berger, M.: Guiding linkages with remote centre of rotation for thermal cutting processes. In: Dede, M. İ. C., İtik, M., Lovasz, E-C. and Kiper, G., Mechanisms, Transmissions and Applications: Proceedings of the Fourth MeTrApp Conference 2007. Springer (2017)

2. Schena B.: Center robotic arm with five-bar spherical linkage for endoscopic camera. International Patent No. WO2007114975. (2007).

3. Yaşır A., Kiper G.: Structural synthesis of 2R1T type mechanisms for minimally invasive surgery applications. In: Dede MIC, İtik M, Lovasz EC, Kiper G (Eds), Mechanisms, Transmissions and Applications: Proceedings of The Fourth Metrapp Conference 2017, pp. 31-38. Springer (2018)

4. Taniguchi, K., Nishikawa, A., Sekimoto, M., Kobayashi, T., Kazuhara, K., Ichihara, T., Kurashita, N., Takiguchi, S., Doki, Y., Mori, M. and Miyazaki, F.: Classification, design and evaluation of endoscope robots. In: Baik, S. H. (Ed.), Robot Surgery. InTech (2010) doi: $10.5772 / 6893$

5. Yaşır, A.: Design of a 2R1T mechanism with remote center of motion for minimally invasive transnasal surgery applications, MSc. thesis, İzmir Institute of Technology, (2018).

6. Herder, J. L.: Energy-free systems; theory, conception and design of statically balanced spring mechanisms. Ph.D. thesis, Delft University of Technology, (2001).

7. Van der Wijk, V., Herder, J. L., Demeulenaere, B.: Comparison of various dynamic balancing principles regarding additional mass and additional inertia. ASME Journal of Mechanisms and Robotics, Vol. 1, Issue 4, November 2009, 04 1006, pp. 1-9 (2009)

8. Wang, J., Gosselin, C. M.: Static balancing of spatial three-degree-of-freedom parallel mechanisms, Mechanism and Machine Theory 34, pp. 437-452. (1999)

9. Wang, J., Gosselin, C. M.: Static balancing of spatial four-degree-of-freedom parallel mechanisms, Mechanism and Machine Theory 35, pp. 563-592. (2000)

10. Van der Wijk, V.: Methodology for analysis and synthesis of inherently force and moment-balanced mechanisms - theory and applications. Dissertation, (2014).

11. Baradat, C., Arakelian, V., Briot, S., Guegan, S.: Design and prototyping of a new balancing mechanism for spatial parallel manipulators, Journal of Mechanical Design, Vol. 130, 072305, (2008)

12. Barents, R., Schenk, M., Van Dorsser, W. D., Wisse, B. M., Herder, J. L.: Spring-to-spring balancing as energy-free adjustment method in gravity equilibrators, ASME Journal of Mechanical Design, Volume 133, Issue 6, pp 061010, (2011) 